\title{
Metabolic Equivalent of Task Unit
}

National Cancer Institute

\section{Source}

National Cancer Institute. Metabolic Equivalent of Task Unit. NCI Thesaurus. Code C127805.

A unit of energy expenditure equal to the ratio of the metabolic rate during physical activity in comparison to a reference metabolic rate. 